\title{
Plasma Membrane Targeting of Protocadherin 15 Is Regulated by the Golgi-Associated Chaperone Protein PIST
}

\author{
Hongyun Nie, ${ }^{1}$ Yueyue Liu, ${ }^{1}$ Xiaolei Yin, ${ }^{2}$ Huiren Cao, ${ }^{1}$ Yanfei Wang, \\ Wei Xiong, ${ }^{3}$ Yushuang Lin, ${ }^{1}$ and Zhigang $\mathrm{Xu}^{1}$ \\ ${ }^{1}$ Shandong Provincial Key Laboratory of Animal Cells and Developmental Biology, Shandong University School of Life Sciences, \\ Jinan, Shandong 250100, China \\ ${ }^{2}$ Taishan Medical University, Taian, Shandong 271000, China \\ ${ }^{3}$ School of Life Sciences, Tsinghua University, Beijing 100084, China
}

Correspondence should be addressed to Yushuang Lin; linyushuang@sdu.edu.cn and Zhigang Xu; xuzg@sdu.edu.cn

Received 18 July 2016; Accepted 26 September 2016

Academic Editor: Renjie Chai

Copyright (c) 2016 Hongyun Nie et al. This is an open access article distributed under the Creative Commons Attribution License, which permits unrestricted use, distribution, and reproduction in any medium, provided the original work is properly cited.

\begin{abstract}
Protocadherin 15 (PCDH15) is a core component of hair cell tip-links and crucial for proper function of inner ear hair cells. Mutations of $P C D H 15$ gene cause syndromic and nonsyndromic hearing loss. At present, the regulatory mechanisms responsible for the intracellular transportation of PCDH15 largely remain unknown. Here we show that PIST, a Golgi-associated, PDZ domaincontaining protein, interacts with PCDH15. The interaction is mediated by the PDZ domain of PIST and the C-terminal PDZ domain-binding interface (PBI) of PCDH15. Through this interaction, PIST retains PCDH15 in the trans-Golgi network (TGN) and reduces the membrane expression of PCDH15. We have previously showed that PIST regulates the membrane expression of another tip-link component, cadherin 23 (CDH23). Taken together, our finding suggests that PIST regulates the intracellular trafficking and membrane targeting of the tip-link proteins $\mathrm{CDH} 23$ and PCDH15.
\end{abstract}

\section{Introduction}

Inner ear hair cells are responsible for mechanoelectrical transduction (MET) that converts mechanical stimuli into electrical signals. MET occurs within stereocilia, the F-actinbased, and microvilli-like protrusions on the apical surface of hair cells [1]. Stereocilia are organized into several rows of increasing heights, forming a staircase-like structure. Several types of extracellular links, including tip-links, topconnectors, lateral-links, ankle-links, and kinociliary-links, connect stereocilia with each other and with the microtubulebased kinocilium [2]. Among these extracellular links, tiplinks are of great importance. Tip-links connect the tip of each stereocilium to the side of its taller neighboring stereocilium and are directly involved in the MET process [3]. The yet unidentified MET channels localize at the lower end of tiplinks and form the so-called MET machinery together with the lower end of tip-links as well as other proteins [4]. When stereocilia are deflected in the excitatory direction, the tension of tip-links increases and the open probability of MET channels also increases, resulting in the influx of cations into hair cells [1].

Protocadherin 15 (PCDH15) is an atypical cadherin that contains eleven extracellular cadherin (EC) repeats, whereas classical cadherins usually have only five EC repeats. Genetic, biochemical, immunochemical, and structural evidence indicated that PCDH15 and cadherin 23 ( $\mathrm{CDH} 23)$, another atypical cadherin, form the lower and higher part of tip-links, respectively [5-7]. PCDH15 and $\mathrm{CDH} 23$ interact with each other via the most $\mathrm{N}$-terminal two $\mathrm{EC}$ repeats and create $\mathrm{a} \sim$ $170 \mathrm{~nm}$ long extracellular link under endolymph-like calcium levels [7-9]. Mutations of $\mathrm{PCDH15}$ gene are responsible for syndromic hearing loss Usher $1 \mathrm{~F}$ or nonsyndromic hearing loss DFNB23 [10-12]. Pcdh15 mutations are also responsible for the hearing and balancing deficits of Ames waltzer (av) mice $[13,14]$. In av mice, stereociliary tip-links are reduced or 


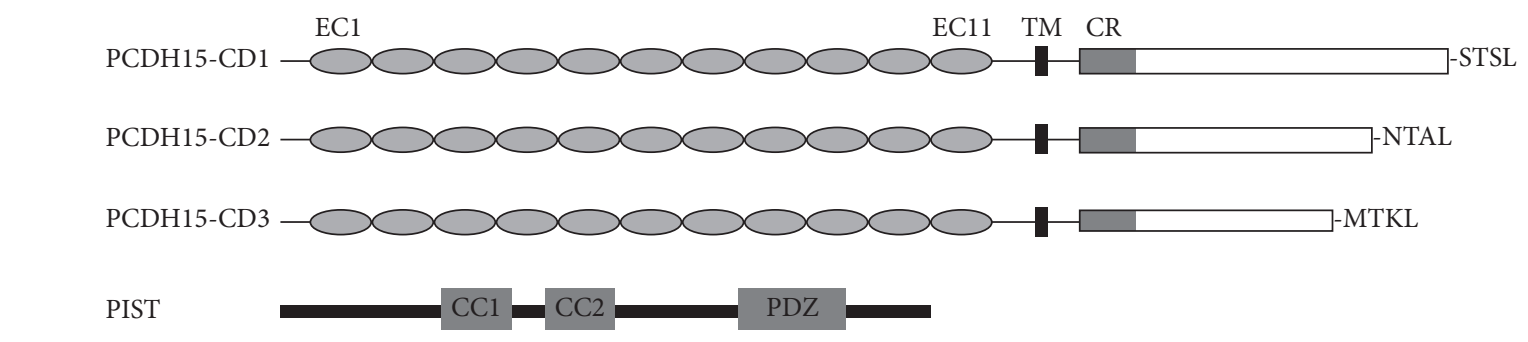

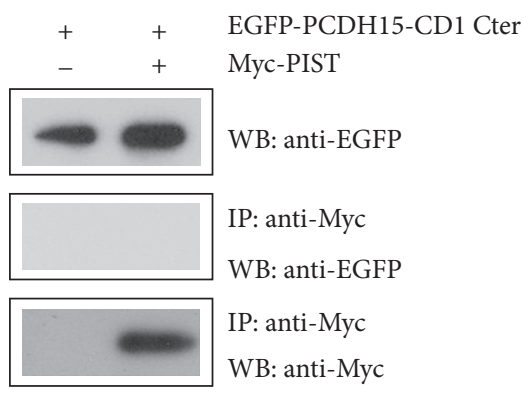

(b)

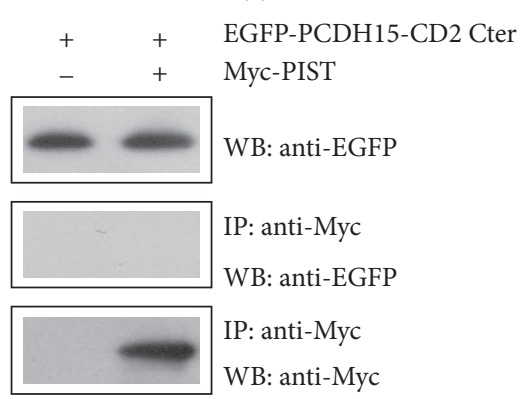

(c)

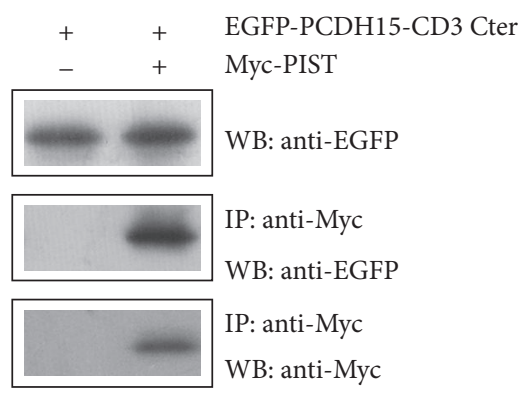

(d)

FIgure 1: PCDH15-CD3 interacts with PIST. (a) Schematic diagram of PCDH15 and PIST domain structure. EC, extracellular cadherin repeats; TM, transmembrane domain; CR, common region; CC1, coiled-coil domain 1; CC2, coiled-coil domain 2; PDZ, PSD-95/discs large/ZO-1 domain. The C-terminal PBI of PCDH15 isoforms (STSL, NTAL, and MTKL) is also indicated. (b) Western blots showing coimmunoprecipitation (co-IP) of EGFP-tagged mouse PCDH15-CD1 cytoplasmic domain with Myc-tagged human PIST. (c) Western blots showing co-IP of EGFP-tagged mouse PCDH15-CD2 cytoplasmic domain with Myc-tagged human PIST. (d) Western blots showing co-IP of EGFP-tagged mouse PCDH15-CD3 cytoplasmic domain with Myc-tagged human PIST. IP indicates antibody used for immunoprecipitation and WB indicates antibody used for detection.

even absent, depending on the nature of the mutations, and hair cells are degenerated eventually, resulting in deafness and vestibular dysfunction [15].

Three prominent PCDH15 isoforms are generated by alternative pre-mRNA splicing, which are PCDH15-CD1, PCDH15-CD2, and PCDH15-CD3 [6]. These PCDH15 isoforms differ in their cytoplasmic domains (Figure 1(a)) and show different spatiotemporal expression pattern in the developing and mature inner ear [6]. Noticeably, different $\mathrm{PCDH} 15$ isoforms contain different PDZ binding interfaces (PBI) at their C-termini, which consist of the last four amino acids and are responsible for binding to PDZ domains. Different PCDH15 isoforms function redundantly during hair cell development, whereas PCDH15-CD2 was shown to be essential for tip-links in mature auditory hair cells $[16,17]$. All PCDH15 isoforms could interact with TMHS (also called LHFPL5), an integral component of MET machinery of cochlear hair cells [18]. PCDH15-CD2, but not PCDH15-CD1 or PCDH15-CD3, also binds TMIE, another MET machinery component [19]. Furthermore, all PCDH15 isoforms directly interact with TMC1 and TMC2, the candidate MET channels, and core component of MET machinery [20,21]. These data suggest that PCDH15 is an important component of the MET machinery.

Several other PCDH15-binding proteins have also been identified so far. PCDH15-CD1 was shown to interact with PDZ domain-containing protein harmonin via its Cterminal PBI $[22,23]$. Further investigation showed that the stereociliary localization of PCDH15 is affected in harmonin knockout mice [24]. PCDH15-CD1 also interacts with Myosin VIIA, and the stereociliary localization of PCDH15 is perturbed in myosin VIIA mutant mice [25]. Recently, PCDH15CD2 was shown to interact with Myosin 3A, which might regulate the transportation of $\mathrm{PCDH} 15$ to stereociliary tips [26]. In the present work, we show that PCDH15-CD3, but not PCDH15-CD1 or PCDH15-CD2, interacts with PDZ domaincontaining protein PIST, which might play an important role in the intracellular trafficking and plasma membrane targeting of PCDH15-CD3.

\section{Materials and Methods}

2.1. DNA Constructs and Antibodies. Chicken Pcdh15-CD3 cDNA was inserted into pBD-GAL4 Cam vector (Stratagene) to express the C-terminal 106 amino acids of chicken PCDH15-CD3 as bait protein. Mouse Pcdh15 cDNAs were inserted into pcDNA3.1(+) to express full length PCDH15 isoforms with a Myc tag between the N-terminal signal peptide and the first EC repeat. Mouse Pcdh15 cDNAs were inserted into pEGFP-C2 or modified pEGFP-C2 (EGFP replaced with $\mathrm{Myc}$ ) to express EGFP-tagged or Myc-tagged PCDH15 cytoplasmic domains. The cDNA encoding mouse PCDH15-CD3 missing the last 4 aa (MTKL) at the Cterminus was inserted into the same vectors to express Myc-PCDH15-CD3 (-MTKL) and Myc-PCDH15-CD3 Cter (-MTKL). Human PIST cDNA was inserted into pEGFP-C2 
or modified pEGFP-C2 to express full length PIST, PIST CC2-plus domain (146-274aa), and PIST PDZ domain (276366aa) as EGFP-fusion or Myc-fusion proteins. Mouse monoclonal anti-EGFP antibody (Cat. number M20004) was from Abmart. Mouse monoclonal anti-Myc antibody (Cat. number M4439) was from Sigma-Aldrich.

2.2. Yeast Two-Hybrid Screen. The yeast two-hybrid screen was performed as previously described [27, 28]. Briefly, yeast strain AH109 (Clontech) was sequentially transformed with the bait plasmid and a chicken cochlear cDNA library in the HybriZAP two-hybrid vector [29]. Totally $2.4 \times 10^{6}$ transformants were selectively screened using HIS3 (at the presence of $2.5 \mathrm{mM}$ of 3-amino-1,2,4-triazole) as the primary reporter gene. The positive colonies were further examined using two more reporter genes ADE2 and lacZ. The prey vectors in triple-positive yeast colonies were recovered and the sequence of cDNA inserts was determined by Sanger sequencing.

2.3. Coimmunoprecipitation (Co-IP). HEK293T cells were transfected with the expression vectors using jetPRIME Transfection Agent (Polyplus, Cat. number PT-114-15) according to the manufacturer's instructions. Transfected cells were washed with phosphate-buffered saline (PBS) 24 hours after transfection and lysed in ice-cold lysis buffer consisting of $150 \mathrm{mM} \mathrm{NaCl}, 50 \mathrm{mM}$ Tris at $\mathrm{pH} 7.5,1 \%$ (vol/vol) Triton X$100,1 \mathrm{mM}$ PMSF, and $1 \times$ protease inhibitor cocktail (Roche). After centrifugation at $4^{\circ} \mathrm{C}$, the supernatant was collected and incubated with immobilized anti-Myc antibody (SigmaAldrich, Cat. number E6654) at $4^{\circ} \mathrm{C}$ overnight. After washing five times with washing buffer (a modified lysis buffer containing $500 \mathrm{mM} \mathrm{NaCl}$ instead of $150 \mathrm{mM}$ ), the immunoprecipitated proteins were separated by polyacrylamide gel electrophoresis (PAGE) and then transferred to PVDF membrane. The blot was incubated with corresponding primary antibodies, followed by incubation with secondary antibodies (Bio-Rad), and the signals were detected with the ECL system (Cell Signaling Technology).

2.4. Immunofluorescence. COS-7 cells were grown on Gelatin-coated glass cover slips and transfected with the expression vectors. Transfected cells were fixed with $4 \%$ paraformaldehyde (PFA) in PBS for 15 minutes and then permeabilized and blocked with PBT1 (0.1\% Triton X-100, 1\% BSA, 5\% heat-inactivated goat serum in PBS, pH 7.3) for 30 minutes, followed by incubation with primary antibody diluted in PBT1 over night at $4^{\circ} \mathrm{C}$. After washing twice with PBT1 for 10 minutes and twice with PBT2 (0.1\% Triton X-100, $0.1 \%$ BSA in PBS) for 5 minutes, cells were incubated with fluorescence-conjugated secondary antibody (Life Tech) in PBT2 for 1 hour, followed by two 5-minute PBT2 washes and two 5-minute PBS washes. For nuclei staining, cells were incubated with DAPI (Gen-View Scientific Inc.) for 15 minutes, followed by four 5-minute PBS washes and then mounted in Glycerol/PBS (1:1). The cells were imaged with a confocal microscope (LSM 700, Zeiss).

\section{Results}

To identify proteins that interact with PCDH15, we performed yeast two-hybrid screens of a chicken cochlear cDNA library using PCDH15 as bait. HIS3 gene was used as the primary reporter gene for the screen in the presence of 3amino-1,2,4-triazole (3-AT) that inhibits the autoactivation of HIS3 reporter gene. When the intact cytoplasmic domain of chicken PCDH15-CD3 was used as bait, it autoactivated the HIS3 reporter gene at the highest 3-AT concentration $(15 \mathrm{mM})$ tested. Then various regions within the PCDH15CD3 cytoplasmic domain were tested for autoactivation. The results showed that the fragment containing the Cterminal 106 amino acids does not activate HIS3 reporter gene at the presence of $2.5 \mathrm{mM} \mathrm{3-AT}$, and this fragment was used in the following yeast two-hybrid screen. Among the positive clones that activate all the three reporter genes (HIS3, ADE2, and lacZ), several clones encode the PDZcontaining, Golgi-associated chaperone protein PIST. PIST has been shown to play important roles in regulating the membrane targeting of several transmembrane proteins such as frizzled, somatostatin receptor subtype 5 (SSTR5) and $\beta 1$ adrenergic receptor ( $\beta 1 \mathrm{AR}$ ) [30-32].

We then performed coimmunoprecipitation (co-IP) experiments to verify the interaction between PCDH15 and PIST. From here on in the rest of our investigation, we focused on mammalian proteins. Our data showed that when overexpressed in HEK293T cells, EGFP-tagged cytoplasmic domain of mouse PCDH15-CD3 was co-IPed with Myctagged human PIST, whereas PCDH15-CD1 and PCDH15CD2 were not (Figures $1(\mathrm{~b})-1(\mathrm{~d})$ ). This result suggests that PCDH15-CD3, but not PCDH15-CD1 or PCDH15-CD2, interacts with PIST.

Previously we have shown that PIST binds to cadherin 23 (CDH23), the upper tip-link component, and retains $\mathrm{CDH} 23$ in the trans-Golgi network (TGN) in cultured cells [33]. To test whether this also applies to PCDH15, we analyzed the subcellular distribution of PCDH15 and PIST in cultured cells by immunofluorescence and confocal microscopy. The results showed that when coexpressed in COS-7 cells, EGFPPIST mainly localizes in the TGN, whereas Myc-PCDH15$\mathrm{CD} 1$ or Myc-PCDH15-CD2 localizes in the cytoplasm as well as on the plasma membrane, consistent with the fact that PCDH15-CD1 or PCDH15-CD2 does not interact with PIST (Figures 2(a) and 2(b)). In contrast, when cotransfected with EGFP-PIST and Myc-PCDH15-CD3, most (70.24\%) cells show colocalization of PCDH15-CD3 with PIST in the TGN (Figure 2(c)).

We then performed co-IP experiments to explore which regions/motifs in PCDH15-CD3 and PIST are responsible for the interaction between them. PIST contains a PDZ domain near the C-terminus and two coiled-coil (CC) domains in the middle part (Figure 3(a)). Co-IP experiments with EGFP-tagged PIST PDZ domain (276-366aa) or the second CC domain (CC2) plus the amino acids between CC2 and PDZ domain (CC2-plus, 146-274aa) revealed that both domains could be co-IPed with Myc-PCDH15-CD3 cytoplasmic domain (Figures 3(b) and 3(c)). PCDH15-CD1, PCDH15-CD2, and PCDH15-CD3 have unique C-termini 

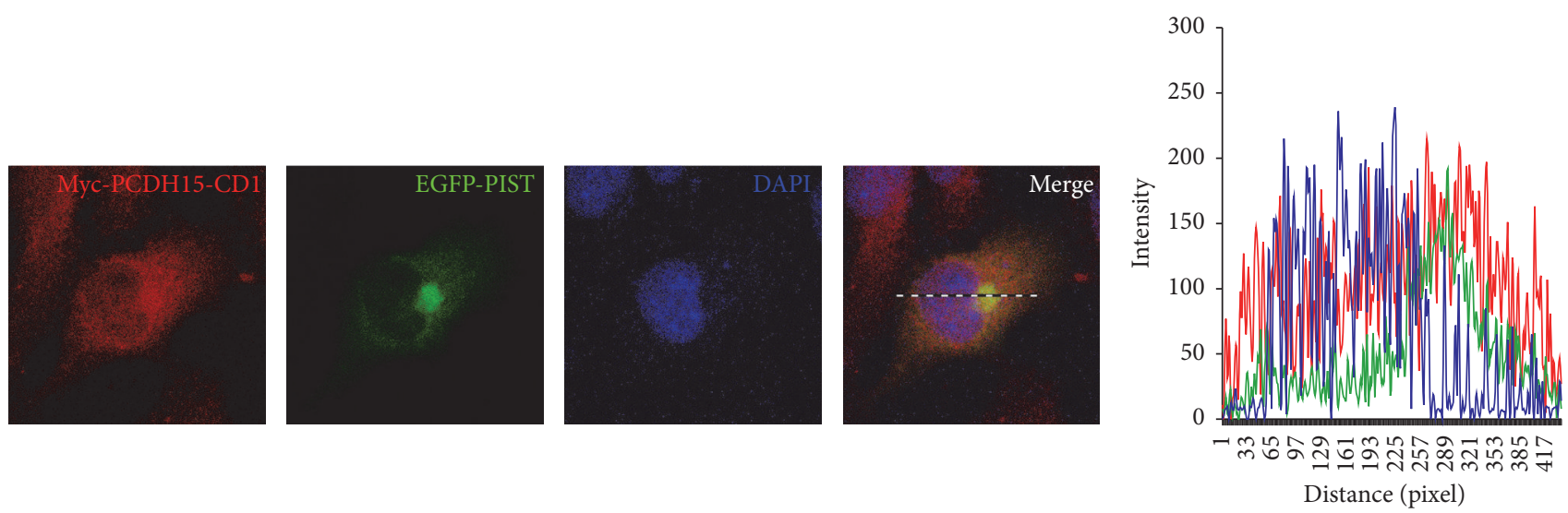

— Myc-PCDH15-CD1

- EGFP-PIST

- DAPI

(a)
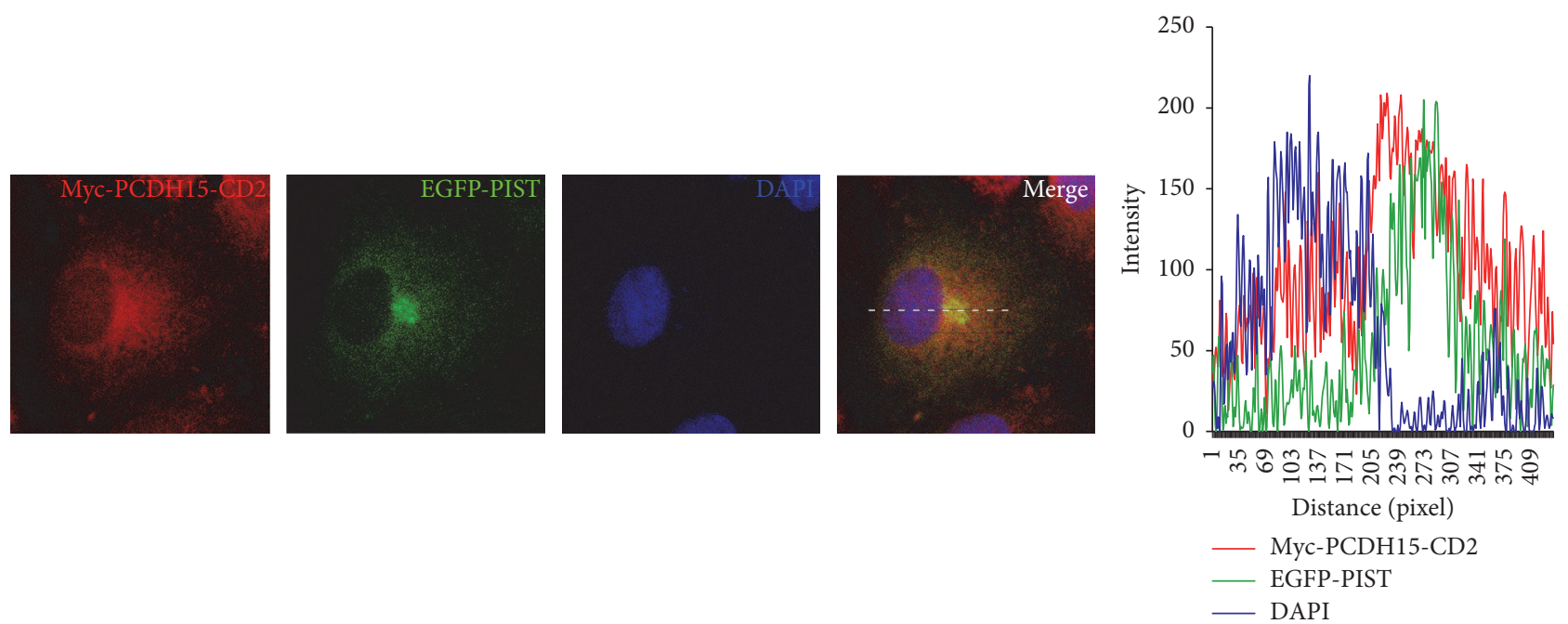

(b)
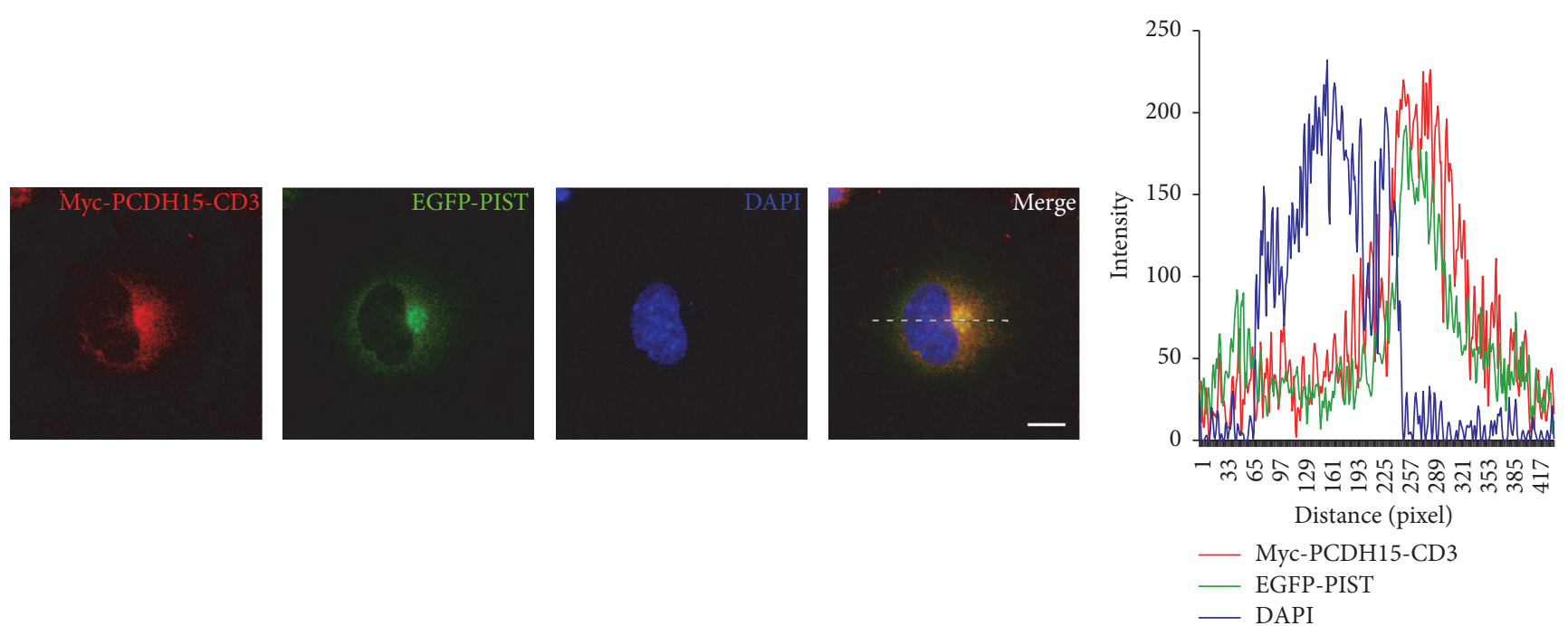

(c)

FIGURE 2: Colocalization of PCDH15-CD3 with PIST in COS-7 cells. (a) In the presence of EGFP-PIST, Myc-PCDH15-CD1 localizes in the cytoplasm as well as on the cell membrane. (b) In the presence of EGFP-PIST, Myc-PCDH15-CD2 localizes in the cytoplasm as well as on the cell membrane. (c) In the presence of EGFP-PIST, Myc-PCDH15-CD3 colocalizes with EGFP-PIST in the TGN. Myc-PCDH15 was stained with mouse anti-Myc antibody and then TRITC-conjugated goat anti-mouse secondary antibody. Nuclei were stained with DAPI. The fluorescent intensity was quantified using Image J. Scale bars, $10 \mu \mathrm{m}$. 


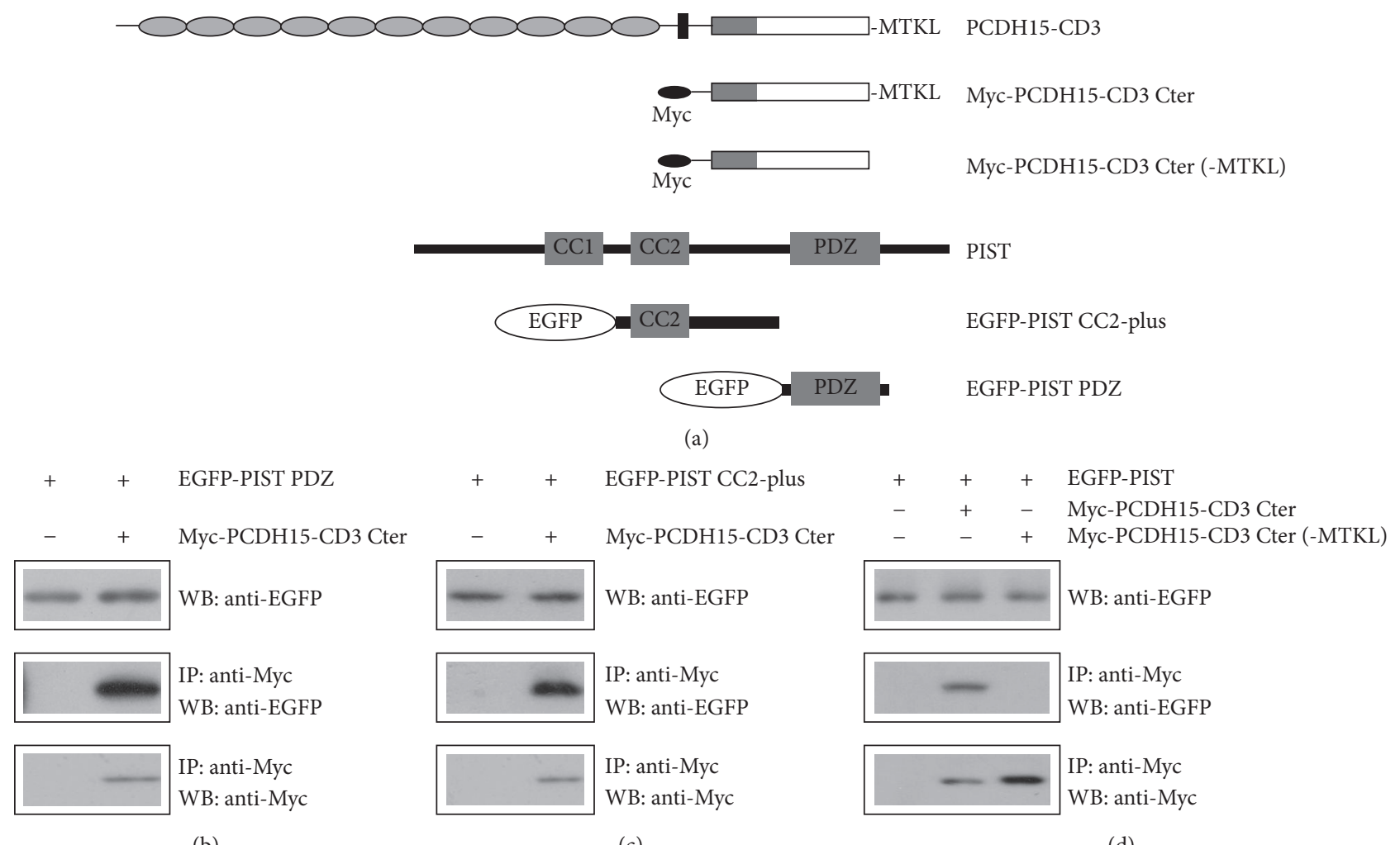

(b)

(c)

(d)

FIgure 3: The C-terminal PBI of PCDH15-CD3 is necessary for the interaction with PIST. (a) Schematic diagram of PCDH15 and PIST domains used in this experiment. (b) Western blots showing coimmunoprecipitation (co-IP) of EGFP-tagged human PIST PDZ domain with Myc-tagged mouse PCDH15-CD3 cytoplasmic domain. (c) Western blots showing co-IP of EGFP-tagged human PIST CC2-plus region with Myc-tagged mouse PCDH15-CD3 cytoplasmic domain. (d) Western blots showing co-IP of EGFP-tagged human PIST with Myc-tagged mouse PCDH15-CD3 cytoplasmic domain with or without the C-terminal PBI (MTKL). IP indicates antibody used for immunoprecipitation and $\mathrm{WB}$ indicates antibody used for detection.

(STSL, NTAL, and MTKL, resp.), which belong to type I PBI [34]. We found that when the C-terminal MTKL of PCDH15CD3 was removed, EGFP-PIST could not be co-IPed with Myc-tagged PCDH15-CD3 cytoplasmic domain anymore (Figure 3(d)). In line with this, Myc-tagged PCDH15-CD3 lacking the C-terminal MTKL is not retained in the TGN by EGFP-PIST anymore (Figures 4(a) and 4(b)). These data suggest that the principal interaction between PCDH15-CD3 and PIST utilizes the PDZ binding interface.

Finally, we examined whether the association with PIST affects the membrane targeting of PCDH15-CD3. When coexpressed with EGFP, Myc-PCDH15-CD3 mainly localizes in the cytoplasm and on the plasma membrane (Figure 5(a)). The plasma membrane localization of Myc-PCDH15-CD3 could be observed more clearly when the extracellular Myc epitope was labeled by anti-Myc antibody without cell permeabilization (Figure $5\left(a^{\prime}\right)$ ). In contrast, when EGFP-PIST is present, Myc-PCDH15-CD3 colocalizes with EGFP-PIST in the TGN, and the membrane labelling in nonpermeabilized cells disappeared completely (Figures $5(b)$ and $5\left(b^{\prime}\right)$ ). These data suggest that PIST retains PCDH15-CD3 in the TGN and reduces the membrane expression of PCDH15-CD3.

\section{Discussion}

PCDH15 and $\mathrm{CDH} 23$ form the lower and upper part of tiplinks, respectively, and are indispensable for hearing transduction. Besides tip-links, PCDH15 and CDH23 also localize at kinociliary-links, transient lateral-links, and ribbon synapse of hair cells $[6,7,35-37]$. It has been suggested that the transportation of PCDH15 to apical (stereociliary bundle) and basal (ribbon synapses) poles depends on distinct trafficking mechanisms. Apically transported PCDH15 was shown to associate with the Arf1-positive early endosomal vesicles and colocalize with the early endosomal marker Rab5, whereas basally transported PCDH15 was shown to associate with AP-1-positive post-trans-Golgi vesicles [38]. The detailed mechanism of PCDH15 transportation remains elusive.

In the present work we show that PIST might play an important role in regulating the intracellular traffic and membrane targeting of PCDH15. Previously we have shown that PIST regulates the membrane expression of $\mathrm{CDH} 23$ [33]. Taken together, our work suggests that PIST regulates the intracellular transportation of both tip-link components, 

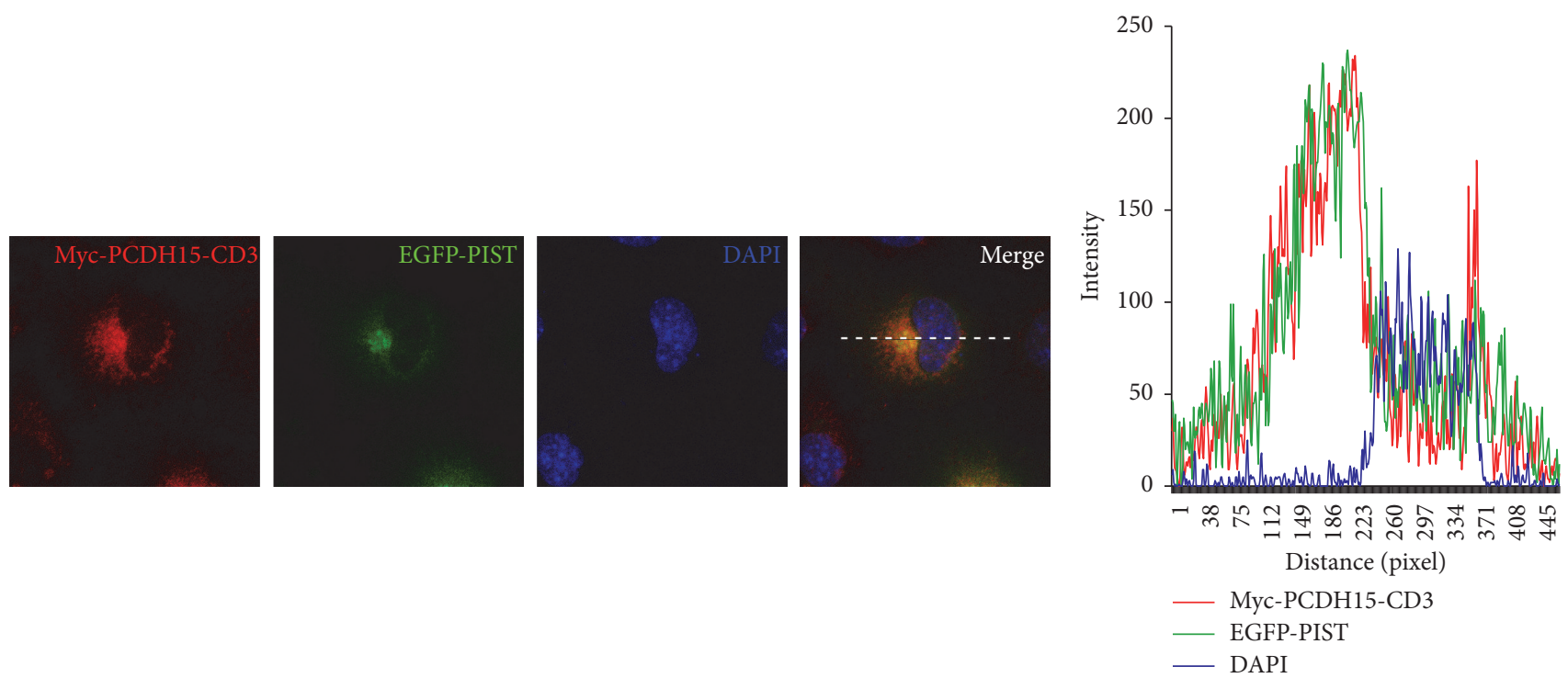

(a)
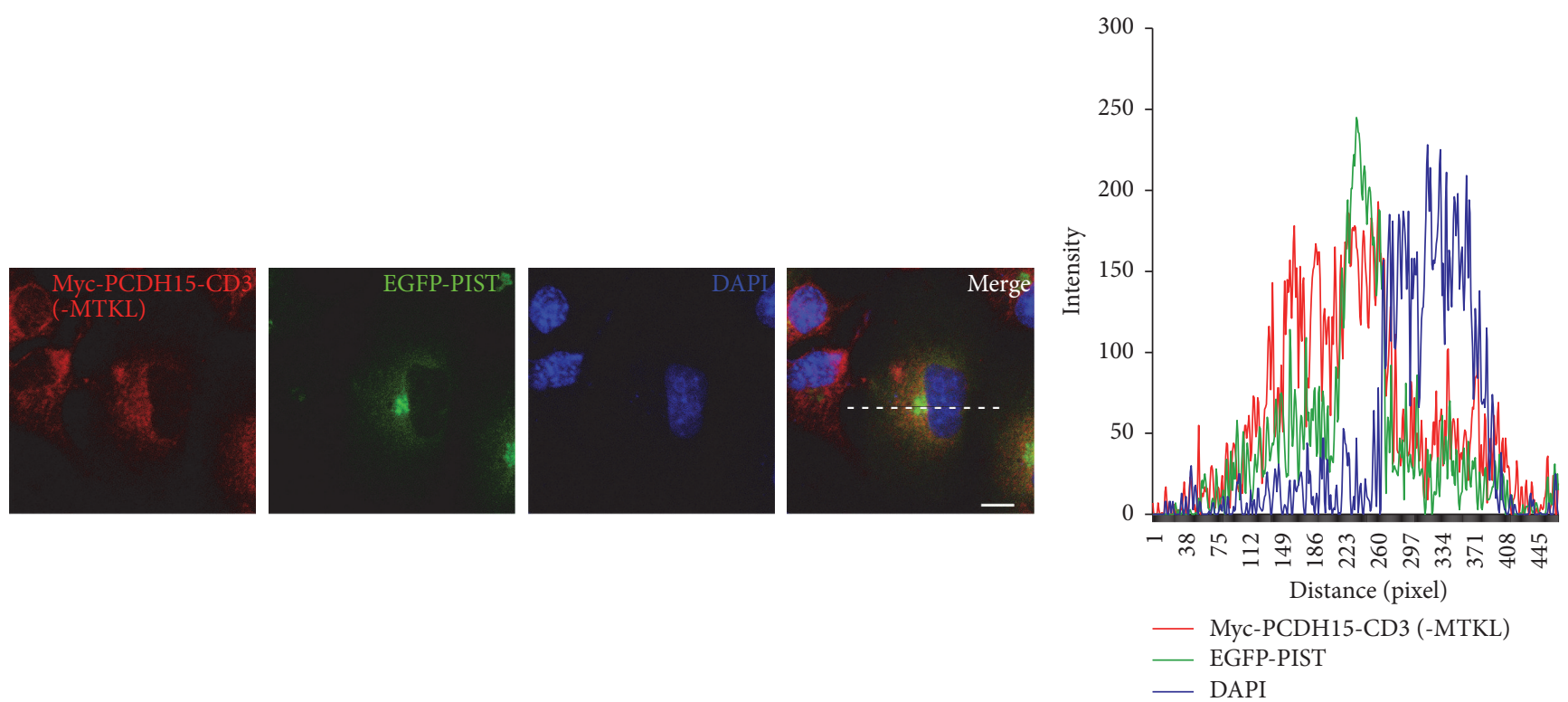

(b)

FIgure 4: The C-terminal PBI of PCDH15-CD3 is necessary for its retention in the TGN of COS-7 cells by PIST. (a) In the presence of EGFP-PIST, Myc-PCDH15-CD3 colocalizes with EGFP-PIST in the TGN. (b) In the presence of EGFP-PIST, Myc-PCDH15-CD3 lacking the C-terminal PBI (MTKL) localizes in the cytoplasm as well as on the cell membrane. Myc-PCDH15 was stained with mouse anti-Myc antibody and then TRITC-conjugated goat anti-mouse secondary antibody. Nuclei were stained with DAPI. The fluorescent intensity was quantified using Image J. Scale bars, $10 \mu \mathrm{m}$.

PCDH15 and CDH23. PIST is a PDZ-containing, Golgiassociated chaperone protein, also named as FIG, GOPC, or CAL. PIST plays important roles in regulating the membrane targeting of transmembrane proteins [30-32]. Notably, PIST was shown to colocalize with the early endosome marker Rab5 and the TGN/endosome marker Rab14 [39]. Our data reveal that PCDH15-CD3 and $\mathrm{CDH} 23$ bind PIST via their $\mathrm{C}$ terminal PBI and colocalize with PIST in the TGN, which might regulate the transportation of $\mathrm{PCDH} 15$ and $\mathrm{CDH} 23$ to the apical surface of hair cells.
Interestingly, we found that, besides the PDZ domain, the CC2 domain and its downstream amino acids (CC2plus) of PIST also interact with PCDH15-CD3. This was also observed in the interaction between PIST and CDH23 [33]. Nevertheless, it seems that PDZ/PBI mediate the principal interaction between PIST and PCDH15-CD3 or $\mathrm{CDH} 23$ because deletion of the $\mathrm{C}$-terminal PBI abolishes the interaction completely. PCDH15-CD1, PCDH15-CD2, and PCDH15-CD3 all contain a type I PBI, but only PCDH15CD3 binds PIST and colocalizes with PIST in the TGN. 

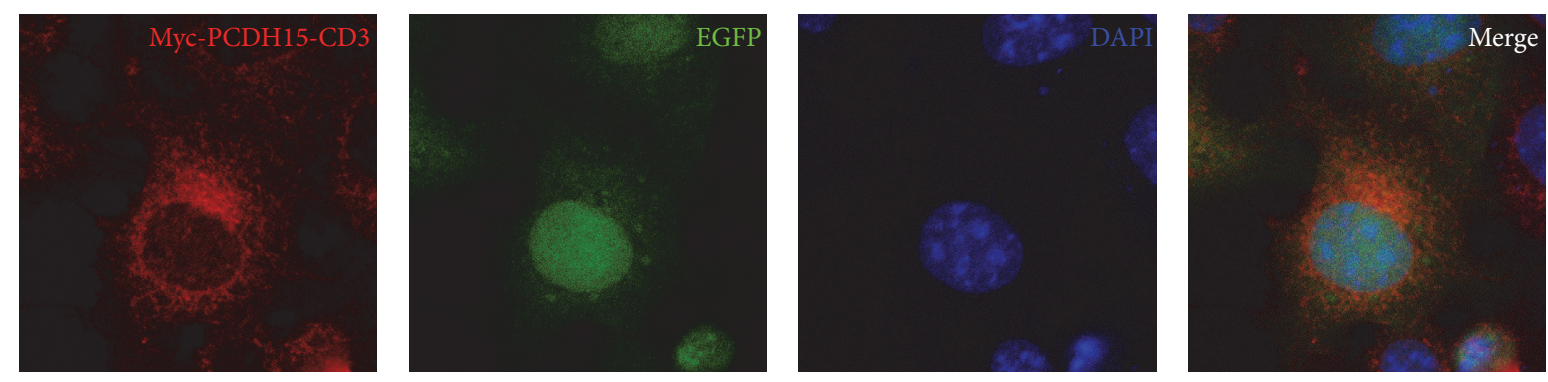

+Triton X-100
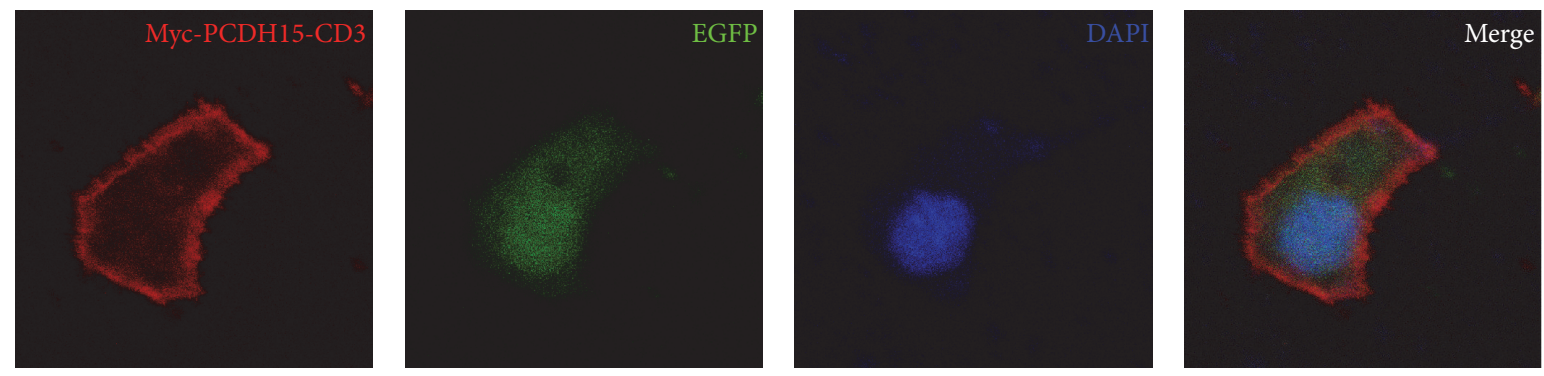

-Triton X-100

$\left(a^{\prime}\right)$
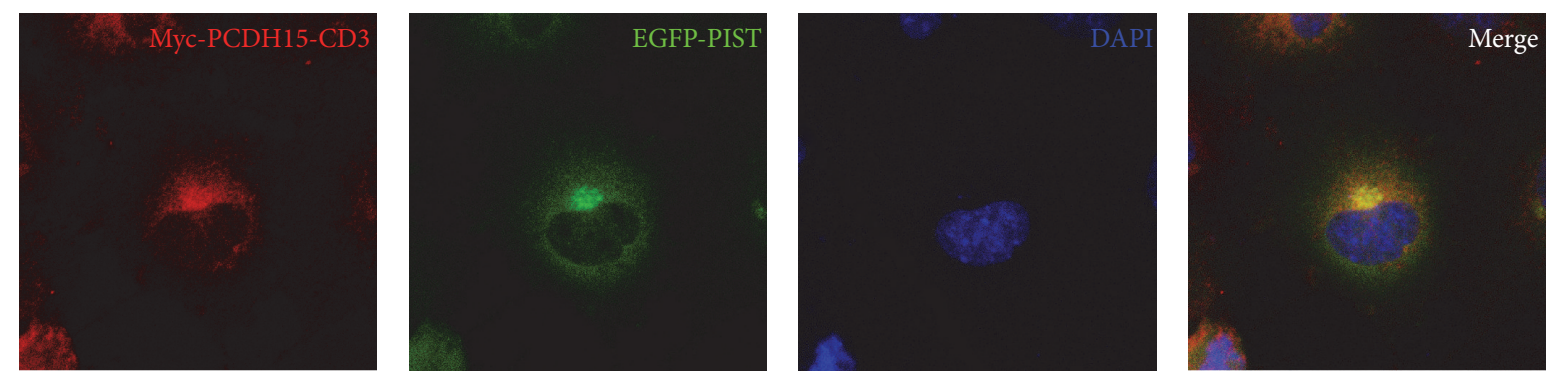

+Triton X-100
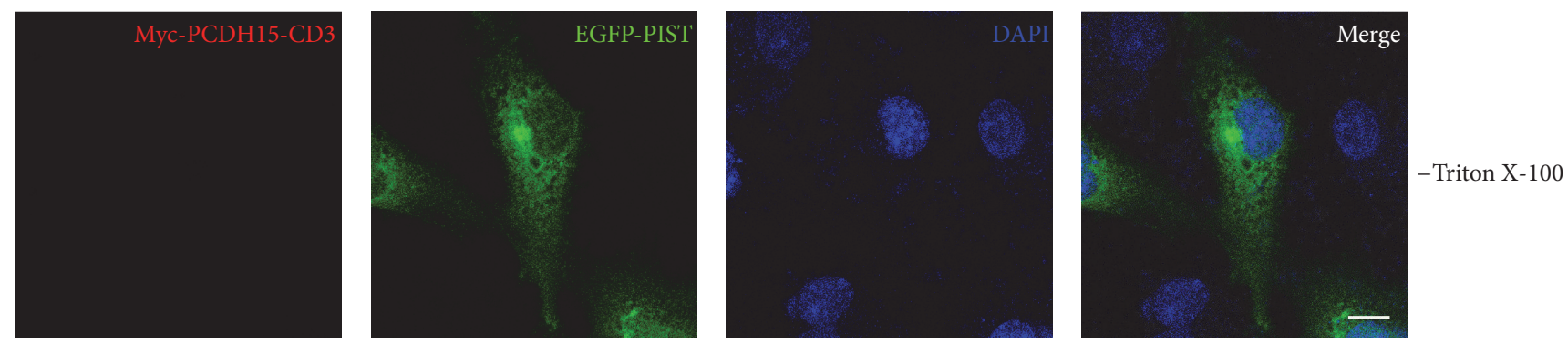

$\left(b^{\prime}\right)$

FIGURE 5: PIST reduces the membrane expression of PCDH15-CD3 in COS-7 cells. (a) Myc-PCDH15-CD3 immunoreactivity shows cytoplasmic and plasma membrane localization when coexpressed with EGFP. (a') Myc-PCDH15-CD3 immunoreactivity using a nonpermeable protocol shows clear plasma membrane localization when coexpressed with EGFP. (b) Myc-PCDH15-CD3 immunoreactivity colocalizes with EGFP-PIST in the TGN. ( $\mathrm{b}^{\prime}$ ) The plasma membrane localization of Myc-PCDH15-CD3 immunoreactivity examined using a nonpermeable protocol disappears when EGFP-PIST is present. For the nonpermeable protocol, staining was performed as normal except that Triton X-100 was excluded. Myc-PCDH15 was stained with mouse anti-Myc antibody and then TRITC-conjugated goat anti-mouse secondary antibody. Nuclei were stained with DAPI. Scale bar, $10 \mu \mathrm{m}$.

Previously, PCDH15-CD1 has been shown to interact with PDZ domain-containing protein harmonin $[22,23]$. The different C-terminal PBI of the three PCDH15 isoforms might mediate interaction with different PDZ domaincontaining proteins and render the PCDH15 isoforms different function or regulation. 


\section{Competing Interests}

The authors declare that there is no conflict of interests regarding the publication of this paper.

\section{Acknowledgments}

The authors thank Dr. W. B. Guggino (Johns Hopkins Medical Institute, Baltimore, MD) for providing the PIST cDNA. This work is supported by grants from the National Basic Research Program of China (2013CB967700 to Zhigang $\mathrm{Xu}$ ), the National Natural Science Foundation of China (31371355 to Zhigang Xu and 31401007 to Yanfei Wang), the China Postdoctoral Science Foundation (2014M560550 to Yanfei Wang), Shandong Provincial Natural Science Foundation (ZR2011CQ034 to Huiren Cao), and the Fundamental Research Funds of Shandong University (2014HW011 to Yanfei Wang).

\section{References}

[1] P. G. Gillespie and U. Müller, "Mechanotransduction by hair cells: models, molecules, and mechanisms," Cell, vol. 139, no. 1, pp. 33-44, 2009.

[2] R. J. Goodyear, W. Marcotti, C. J. Kros, and G. P. Richardson, "Development and properties of stereociliary link types in hair cells of the mouse cochlea," Journal of Comparative Neurology, vol. 485, no. 1, pp. 75-85, 2005.

[3] J. A. Assad, G. M. Shepherd, and D. P. Corey, "Tip-link integrity and mechanical transduction in vertebrate hair cells," Neuron, vol. 7, no. 6, pp. 985-994, 1991.

[4] M. Beurg, R. Fettiplace, J.-H. Nam, and A. J. Ricci, "Localization of inner hair cell mechanotransducer channels using highspeed calcium imaging," Nature Neuroscience, vol. 12, no. 5, pp. 553-558, 2009.

[5] J. Siemens, C. Lillo, R. A. Dumont et al., "Cadherin 23 is a component of the tip link in hair-cell stereocilla," Nature, vol. 428, no. 6986, pp. 950-955, 2004.

[6] Z. M. Ahmed, R. Goodyear, S. Riazuddin et al., "The tip-link antigen, a protein associated with the transduction complex of sensory hair cells, is protocadherin-15," The Journal of Neuroscience, vol. 26, no. 26, pp. 7022-7034, 2006.

[7] P. Kazmierczak, H. Sakaguchi, J. Tokita et al., "Cadherin 23 and protocadherin 15 interact to form tip-link filaments in sensory hair cells," Nature, vol. 449, no. 7158, pp. 87-91, 2007.

[8] D. N. Furness, Y. Katori, B. Nirmal Kumar, and C. M. Hackney, "The dimensions and structural attachments of tip links in mammalian cochlear hair cells and the effects of exposure to different levels of extracellular calcium," Neuroscience, vol. 154, no. 1, pp. 10-21, 2008.

[9] M. Sotomayor, W. A. Weihofen, R. Gaudet, and D. P. Corey, "Structure of a force-conveying cadherin bond essential for inner-ear mechanotransduction," Nature, vol. 492, no. 7427, pp. 128-132, 2012.

[10] Z. M. Ahmed, S. Riazuddin, S. L. Bernstein et al., "Mutations of the protocadherin gene PCDH15 cause usher syndrome type 1F,' The American Journal of Human Genetics, vol. 69, no. 1, pp. 25-34, 2001.

[11] K. N. Alagramam, H. Yuan, M. H. Kuehn et al., "Mutations in the novel protocadherin PCDH15 cause Usher syndrome type
1F,' Human Molecular Genetics, vol. 10, no. 16, pp. 1709-1718, 2001.

[12] Z. M. Ahmed, S. Riazuddin, J. Ahmad et al., "PCDH15 is expressed in the neurosensory epithelium of the eye and ear and mutant alleles are responsible for both USH1F and DFNB23," Human Molecular Genetics, vol. 12, no. 24, pp. 3215-3223, 2003.

[13] K. N. Alagramam, C. L. Murcia, H. Y. Kwon, K. S. Pawlowski, C. G. Wright, and R. P. Woychik, "The mouse Ames waltzer hearing-loss mutant is caused by mutation of Pcdh15, a novel protocadherin gene," Nature Genetics, vol. 27, no. 1, pp. 99-102, 2001.

[14] Q. Y. Zheng, H. Yu, J. L. Washington III et al., "A new spontaneous mutation in the mouse protocadherin 15 gene," Hearing Research, vol. 219, no. 1-2, pp. 110-120, 2006.

[15] K. N. Alagramam, R. J. Goodyear, R. Geng et al., "Mutations in protocadherin 15 and cadherin 23 affect tip links and mechanotransduction in mammalian sensory hair cells," PLoS ONE, vol. 6, no. 4, Article ID e19183, 2011.

[16] S. W. Webb, N. Grillet, L. R. Andrade et al., "Regulation of $\mathrm{PCDH} 15$ function in mechanosensory hair cells by alternative splicing of the cytoplasmic domain," Development, vol. 138, no. 8, pp. 1607-1617, 2011.

[17] E. Pepermans, V. Michel, R. Goodyear et al., “The CD2 isoform of protocadherin-15 is an essential component of the tiplink complex in mature auditory hair cells," EMBO Molecular Medicine, vol. 6, no. 7, pp. 984-992, 2014.

[18] W. Xiong, N. Grillet, H. M. Elledge et al., "TMHS is an integral component of the mechanotransduction machinery of cochlear hair cells," Cell, vol. 151, no. 6, pp. 1283-1295, 2012.

[19] B. Zhao, Z. Wu, N. Grillet et al., "TMIE is an essential component of the mechanotransduction machinery of cochlear hair cells," Neuron, vol. 84, no. 5, pp. 954-967, 2014.

[20] R. Maeda, K. S. Kindt, W. Mo et al., “Tip-link protein protocadherin 15 interacts with transmembrane channel-like proteins TMC1 and TMC2," Proceedings of the National Academy of Sciences of the United States of America, vol. 111, no. 35, pp. 12907-12912, 2014.

[21] M. Beurg, W. Xiong, B. Zhao, U. Müller, and R. Fettiplace, "Subunit determination of the conductance of hair-cell mechanotransducer channels," Proceedings of the National Academy of Sciences of the United States of America, vol. 112, no. 5, pp. 15891594, 2015.

[22] A. Adato, V. Michel, Y. Kikkawa et al., "Interactions in the network of Usher syndrome type 1 proteins," Human Molecular Genetics, vol. 14, no. 3, pp. 347-356, 2005.

[23] J. Reiners, T. Märker, K. Jürgens, B. Reidel, and U. Wolfrum, "Photoreceptor expression of the Usher syndrome type 1 protein protocadherin 15 (USH1F) and its interaction with the scaffold protein harmonin (USH1C)," Molecular Vision, vol. 11, pp. 347355, 2005.

[24] D. Yan, K. Kamiya, X. M. Ouyang, and X. Z. Liu, "Analysis of subcellular localization of Myo7a, Pcdh15 and Sans in Ushlc knockout mice," International Journal of Experimental Pathology, vol. 92, no. 1, pp. 66-71, 2011.

[25] M. Senften, M. Schwander, P. Kazmierczak et al., "Physical and functional interaction between protocadherin 15 and myosin VIIa in mechanosensory hair cells," The Journal of Neuroscience, vol. 26, no. 7, pp. 2060-2071, 2006.

[26] M. Grati, D. Yan, M. H. Raval et al., "MYO3A causes human dominant deafness and interacts with protocadherin 15-CD2 isoform," Human Mutation, vol. 37, no. 5, pp. 481-487, 2016. 
[27] Z. Xu, A. W. Peng, K. Oshima, and S. Heller, "MAGI-1, a candidate stereociliary scaffolding protein, associates with the tiplink component cadherin 23," Journal of Neuroscience, vol. 28, no. 44, pp. 11269-11276, 2008.

[28] H. Cao, X. Yin, Y. Cao et al., "FCHSD1 and FCHSD2 are expressed in hair cell stereocilia and cuticular plate and regulate actin polymerization in vitro," PLoS ONE, vol. 8, no. 2, Article ID e56516, 2013.

[29] S. Heller, C. A. Sheane, Z. Javed, and A. J. Hudspeth, "Molecular markers for cell types of the inner ear and candidate genes for hearing disorders," Proceedings of the National Academy of Sciences of the United States of America, vol. 95, no. 19, pp. 1140011405, 1998.

[30] R. Yao, T. Maeda, S. Takada, and T. Noda, "Identification of a PDZ domain containing golgi protein, GOPC, as an interaction partner of frizzled," Biochemical and Biophysical Research Communications, vol. 286, no. 4, pp. 771-778, 2001.

[31] W. Wente, T. Stroh, A. Beaudet, D. Richter, and H.-J. Kreienkamp, "Interactions with PDZ domain proteins PIST/ GOPC and PDZK1 regulate intracellular sorting of the somatostatin receptor subtype 5," The Journal of Biological Chemistry, vol. 280, no. 37, pp. 32419-32425, 2005.

[32] J. Koliwer, M. Park, C. Bauch, M. Von Zastrow, and H.J. Kreienkamp, "The golgi-associated PDZ domain protein PIST/GOPC stabilizes the $\beta 1$-Adrenergic receptor in intracellular compartments after internalization," Journal of Biological Chemistry, vol. 290, no. 10, pp. 6120-6129, 2015.

[33] Z. Xu, K. Oshima, and S. Heller, "PIST regulates the intracellular trafficking and plasma membrane expression of cadherin 23," BMC Cell Biology, vol. 11, article 80, 2010.

[34] M. Sheng and C. Sala, "PDZ domains and the organization of supramolecular complexes," Annual Review of Neuroscience, vol. 24, pp. 1-29, 2001.

[35] V. Michel, R. J. Goodyear, D. Weil et al., "Cadherin 23 is a component of the transient lateral links in the developing hair bundles of cochlear sensory cells," Developmental Biology, vol. 280, no. 2, pp. 281-294, 2005.

[36] A. Lagziel, Z. M. Ahmed, J. M. Schultz, R. J. Morell, I. A. Belyantseva, and T. B. Friedman, "Spatiotemporal pattern and isoforms of cadherin 23 in wild type and waltzer mice during inner ear hair cell development," Developmental Biology, vol. 280, no. 2, pp. 295-306, 2005.

[37] M. Zallocchi, D. T. Meehan, D. Delimont et al., "Role for a novel Usher protein complex in hair cell synaptic maturation," PLoS ONE, vol. 7, no. 2, Article ID e30573, 2012.

[38] M. Zallocchi, D. Delimont, D. T. Meehan, and D. Cosgrove, "Regulated vesicular trafficking of specific PCDH15 and VLGR1 variants in auditory hair cells," Journal of Neuroscience, vol. 32, no. 40, pp. 13841-13859, 2012.

[39] R. Lu, L. Stewart, and J. M. Wilson, "Scaffolding protein GOPC regulates tight junction structure," Cell and Tissue Research, vol. 360, no. 2, pp. 321-332, 2015. 

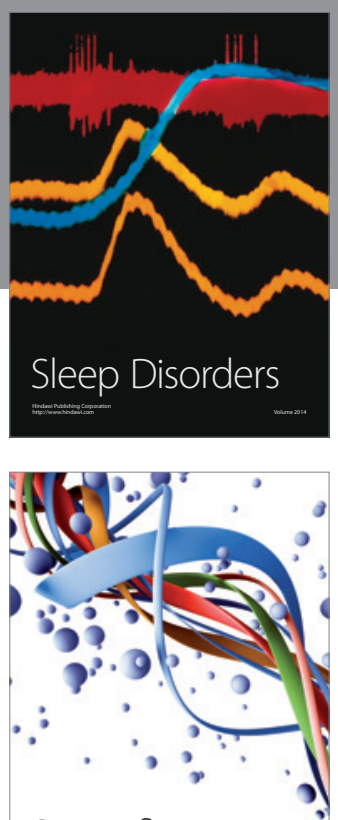

Scientifica
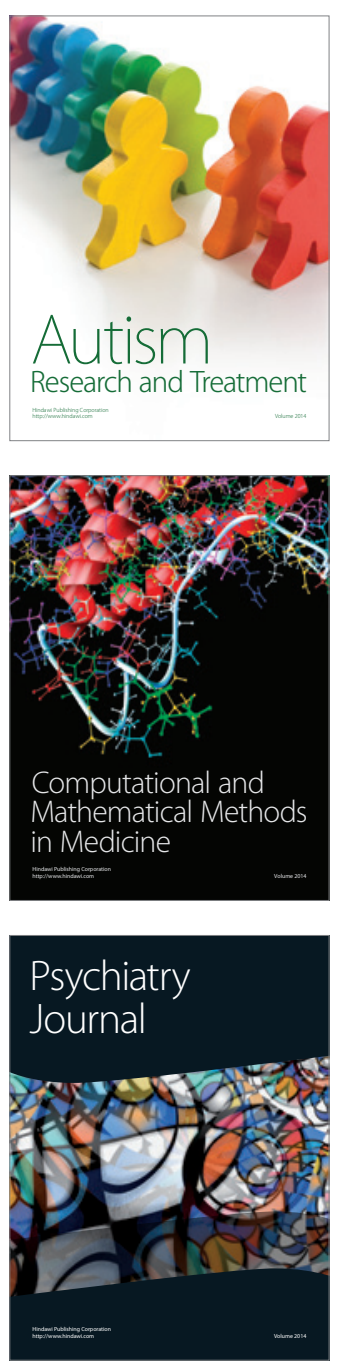
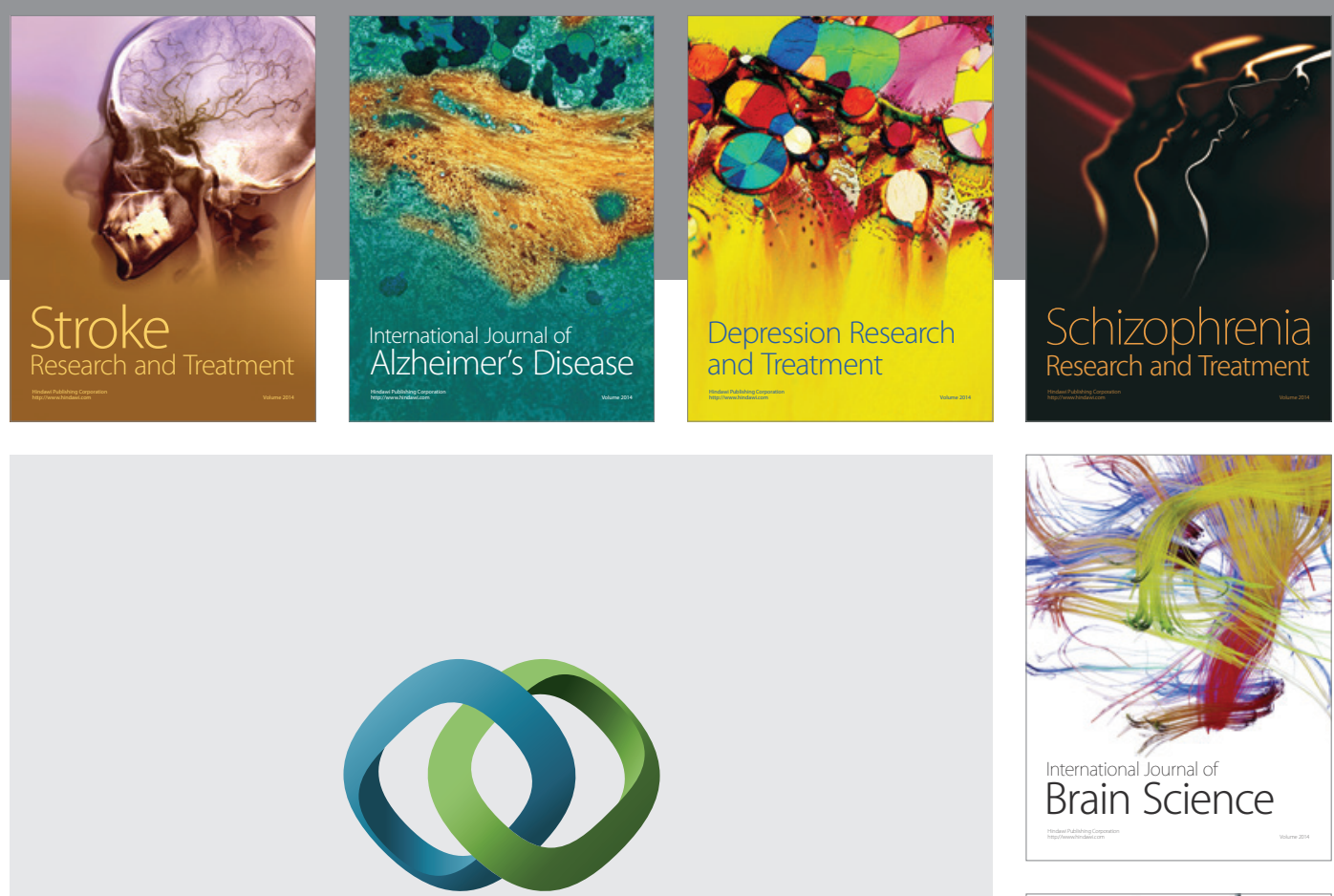

\section{Hindawi}

Submit your manuscripts at

http://www.hindawi.com
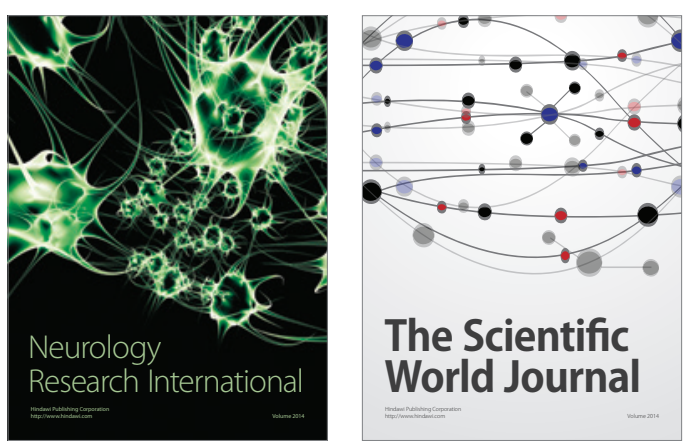

The Scientific World Journal

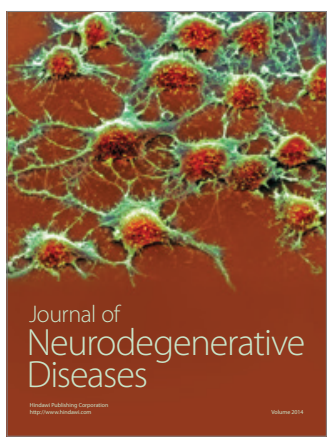

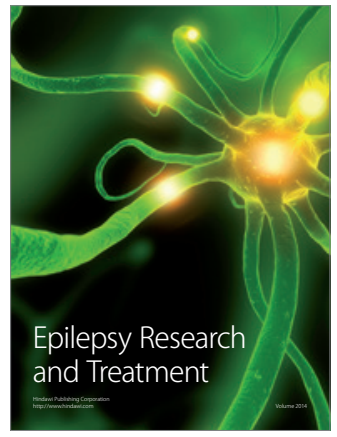

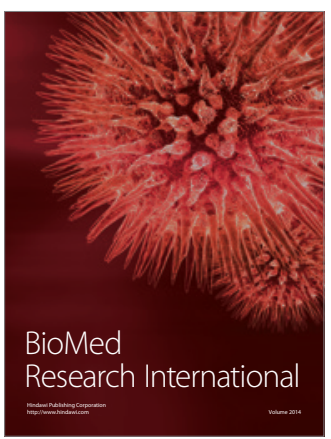

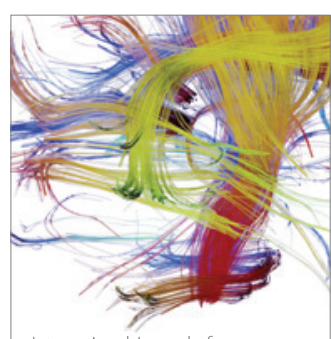

Brain Science

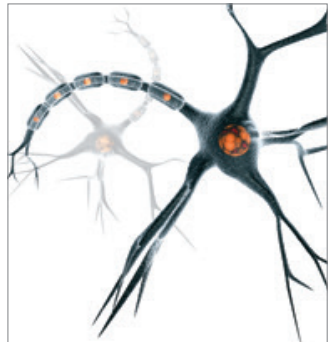

Neural Plasticity
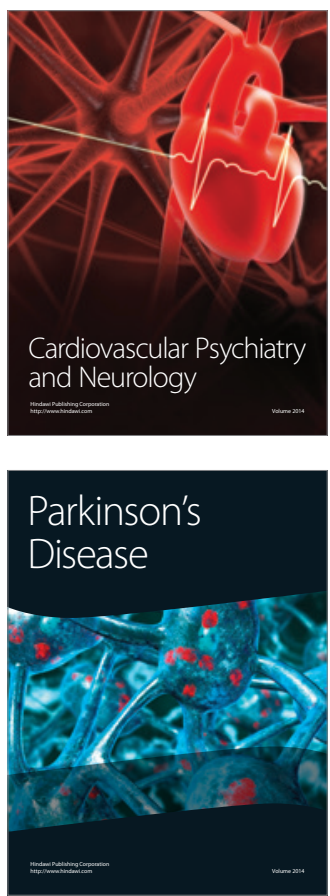